\title{
D'où vient la phytothérapie et vers où tend-elle ?
}

\author{
P. Goetz \\ (C) Lavoisier SAS 2021
}

À l'occasion d'une lecture de soins donnés par les Égyptiens de l'Antiquité à des troubles gynécologiques, j'ai eu l'occasion de d'ouvrir une page de traduction du papyrus Ebers. Ce papyrus contient des détails pour établir un diagnostic mais aussi des modes de traitement dont ceux avec des plantes de cette région que l'on retrouve encore aujourd'hui. Avant de lire et comprendre une formulation de l'Antiquité il faut comprendre ou savoir quelle plante est citée par tel mot latin, grec ou égyptien. En égyptien ancien la langue s'écrit avec des consonnes et il s'agit d'y remettre des voyelles qui peuvent alors rapprocher les noms reconstitués avec des noms de plantes du copte ou du grec ancien, éventuellement d'une langue sémitique. Grâce à Pedanios Dioscorides une longue liste de plantes nous a été transmise [1] et bien étudiée par différents spécialistes comme Goodyer et Gunther [2]. La médecine égyptienne, si elle fut importante est cependant mal connue aujourd'hui. On n'est pas sûr qu'elle avait adopté les théories des humeurs des gréco-latins qui a faussement guidé la médecine arabe et la médecine européenne jusqu'à la fin du XIX ${ }^{\mathrm{e}}$ siècle.

La lecture de la formule du papyrus Ebers, pEbers 802. $94,16 c-17 b$, nous donne la formulation suivante qui a été expliquée par Jean [2] (Fig. 1).

${ }^{16 \mathrm{c}}$ Autre (médication) : ${ }^{16 \mathrm{~d}}$ (fruit de la) plante-bebes (a) : $\mathbf{1}$; résine de térébinthe $(\mathrm{b}): \mathbf{1}$; résine-hedjou $(\mathrm{c}): 1$; bièredjeseret (d) : 1 ;

${ }^{17}$ a akènes frais d'arroche (e) : $\mathbf{1}$; crottes de mouches (f) : $\mathbf{1}$.

${ }^{17 b}$ Préparer sous forme d'un ovule (g), et introduire dans son vagin (h).

« Fruit de la plante besbes

Résine de térébinthe $s n \underline{t} r$

Résine $h \underline{d} w$

Bière djeseret

Akènes frais d'arroche

Crottes de mouches

\section{P. Goetz $(\bowtie)$}

DU de phytothérapie, Paris-XIII,

F-93017 Bobigny cedex, France

e-mail : goetz.correpondance@gmail.com
Préparation sous forme d'ovule $(j r(=w) m m t)$

À introduire dans le vagin de la malade »

Le besbes n'est pas reconnu dans la liste égyptienne des noms de plantes, mais est probablement celle qui s'appelle en arabe basbas et qui est le fenouil (Fœniculum vulgare Mill.). Les graines de la plante-besbes ont une activité ocytocique [2].

Le sntrr (ou sonter) est en général le terme qui signifie encens ou une substance odorifiante utilisée dans les lieux de culte, mais Jean (égyptologue) estime qu'il s'agit de la résine de Pistacia terebinthinum. Ce sntr est connu pour ses capacités antiseptiques, anti-inflammatoires, antiœdémateuses, ou aussi une activité cortisone-like par stimulation de l'axe hypophyso-corticosurrénalien, et un effet calmant.

La résine hạdw est interprètée par Jean comme la résine hedjou qui est une résine blanche ou une résine de plante blanche mais signifie aussi lait. ( $h \underline{d} \underline{t}=$ substance blanche dans la Chapelle de calcite de Thoutmosis IV). Cette résine a une action stimulante, diurétique et diaphorétique.

La bière djeseret est une bière blonde qui ici aurait été traitée spécialement avec une plante qui lui renforçait le goût (en opposition à la bière brune kenemes) [2].

Les akènes d'arroche des jardins (Atriplex hortensis) que Dioscoride [2] dit s'appeler asonteri chez les Égyptiens. On en faisait de la farine. Elle contient des électrolytes et aurait un effet cardiotonique.

Les crottes de mouche devaient avoir une certaine importance pour l'apothicaire égyptien mais dont l'intérêt nous reste inconnu.

Ce mélange en intravaginal sous forme d'ovule devait s'introduire dans le vagin. Elle peut être utilisée en cas de pré-éclampsie mais aussi lors d'aménorrhée.

Les constituants peuvent avoir des effets cardiovasculaires, mais aussi anti-inflammatoire. Par ses constituants elle pourrait agir en stimulant les parties génitales de la femme dans l'espoir de faire venir les règles.

En conclusion, la médecine égyptienne révélée par le papyrus Ebers semble assez moderne par rapport à la médecine gréco-latine qui intoxiqua la pseudo-science médicale européenne. 


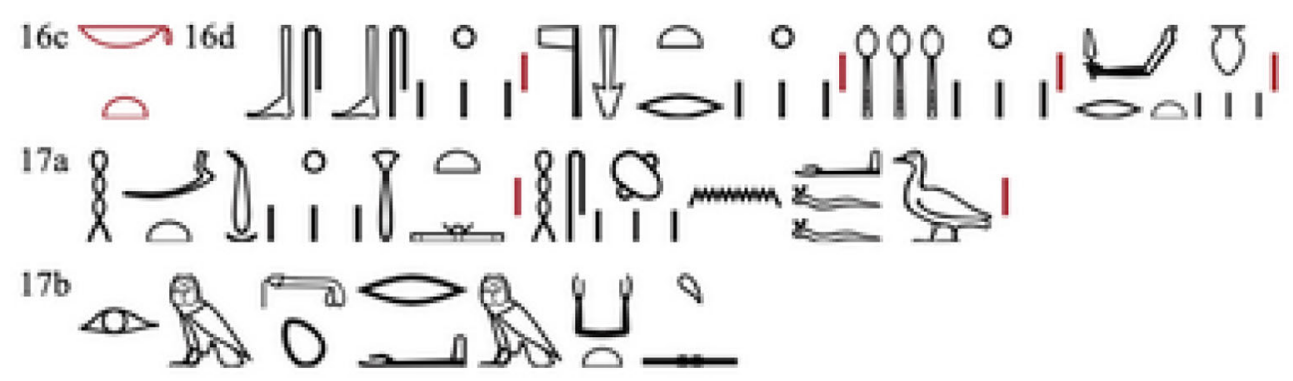

Fig. 1 Papyrus Ebers, pEbers 802. 94,16c-17b

Elle nous rappelle, qu'en dehors du traitement de drainage et de terrain, un peu spécifique à la phytothérapie française, le traitement par les plantes (quand les effets de ses constituants chimiques sont connus) repose sur la physiopathologie de la maladie à traiter. La phytothérapie n'est pas simplement une allopathie, elle répond à des processus pathologiques et non à des symptômes. Cette physiopathologie elle-même devient de plus en plus précise en s'enrichissant des données génétiques et immunologiques récentes. C'est la démarche qui est poursuivie dans cette revue qui doit être un guide dans le parcours de la thérapie par les plantes médicinales.

\section{Références}

1. Manniche L. (2006) An ancient egyptian herbal. British Museum Press

2. Dioscorides P., Gabaudan F. C. (2006) Dioscórides. Estudios y traducción, Universidad de Salamanca, Jan 12006

3. Jean R.-A. (2020) Clinique obstétricale égyptienne - XL . Les accouchements divins, royaux et humains (6). Les textes (4). Les Textes médicaux (2). In : Histoire de la médecine en Égypte ancienne, Paris, 28 février 2020. http://medecineegypte.canalblog. com/pages/obstetrique\%2D \%2D\%2Dx1/38062850.html

4. Jean R. A., Loyrette A-M, et al (2020) Cahiers intégrés de médecine égyptienne (CIME), VII, 1, Paris - Le Caire 